\title{
Admissible closures of polynomial time computable arithmetic
}

\author{
Dieter Probst • Thomas Strahm
}

Received: 9 September 2010 / Accepted: 19 April 2011 / Published online: 4 May 2011

(C) Springer-Verlag 2011

\begin{abstract}
We propose two admissible closures $\mathbb{A}(\mathrm{PTCA})$ and $\mathbb{A}(\mathrm{PHCA})$ of Ferreira's system PTCA of polynomial time computable arithmetic and of full bounded arithmetic (or polynomial hierarchy computable arithmetic) PHCA. The main results obtained are: (i) $\mathbb{A}(\mathrm{PTCA})$ is conservative over PTCA with respect to $\forall \exists \Sigma_{1}^{b}$ sentences, and (ii) $\mathbb{A}(\mathrm{PHCA})$ is conservative over full bounded arithmetic PHCA for $\forall \exists \Sigma_{\infty}^{b}$ sentences. This yields that (i) the $\Sigma_{1}^{b}$ definable functions of $\mathbb{A}(\mathrm{PTCA})$ are the polytime functions, and (ii) the $\Sigma_{\infty}^{b}$ definable functions of $\mathbb{A}(\mathrm{PHCA})$ are the functions in the polynomial time hierarchy.
\end{abstract}

Keywords Polynomial time computable arithmetic - Kripke Platek set theory · Second order arithmetic

\section{Introduction}

The theory of admissible sets, i.e. Kripke-Platek set theory, is one of the most familiar subsystems of Zermelo-Fraenkel set theory. Apart from their significance for definability theory, theories for (iterated) admissible sets have long been central to proof theory, see Jäger [12,13] and Pohlers [14].

D. Probst

Mathematisches Institut, Ludwig-Maximilians-Universität München, Theresienstrasse 39, 80333 München, Germany

e-mail: Dieter.Probst@mathematik.uni-muenchen.de; dieter.probst@gmail.com

T. Strahm (凶)

Institut für Informatik und Angewandte Mathematik, Universität Bern, Neubrückstrasse 10, 3012 Bern, Switzerland e-mail: strahm@iam.unibe.ch

URL: http://www.iam.unibe.ch/ strahm 
The paper is concerned with systems of Kripke-Platek set theory which are prooftheoretically weak. It can be seen as a companion to Jäger's KPu ${ }^{r}$ of Kripke-Platek set theory with the natural numbers as urelements, which is a conservative extension of Peano arithmetic PA, cf. Jäger [13]. Whereas in $\mathrm{KPu}^{r}$ the axioms of admissible sets are stated above the ground theory PA, this paper deals with similar theories above versions of bounded arithmetic, namely Ferreira's polynomial time computable arithmetic PTCA and the theory PHCA of polynomial hierarchy computable arithmetic corresponding to full bounded arithmetic $\Sigma_{\infty}^{b}-\mathrm{NIA}$, cf. Ferreira $[8,9]$.

In contrast to the theory $\mathrm{KPu}^{r}$, we no longer claim that the collection of urelements forms a set, since the presence of $\Delta_{0}$ separation would immediately yield full unbounded quantification over the urelements. With respect to our urelements $W$ (the collection of binary words), we study two set existence principles for collections of words, namely:

\section{(W.0) The collection of all subwords of a given binary word forms a set;}

\section{(W.1) The collection of all words whose length is less than or equal to the length of a given binary word forms a set.}

Based on the two set existence principles (W.0) and (W.1), we study two admissible closures of polynomial time computable arithmetic PTCA. The first closure, A (PTCA), extends PTCA by (W.0) and the usual axioms of Kripke-Platek set theory, namely pairing, union, $\Delta_{0}$ separation and $\Delta_{0}$ collection, as well as foundation in the form of the regularity axiom and induction along the binary words $\mathrm{W}$ for $\Delta_{0}$ formulas. The second closure, $\mathbb{A}(\mathrm{PHCA})$, is obtained from $\mathbb{A}(\mathrm{PTCA})$ by replacing (W.0) by the stronger axiom (W.1). It will be seen that $\mathbb{A}(\mathrm{PHCA})$ directly contains full bounded arithmetic PHCA.

In this paper we will establish that $\mathbb{A}(\mathrm{PTCA})$ is conservative over PTCA with respect to $\forall \exists \Sigma_{1}^{b}$ sentences and $\mathbb{A}(\mathrm{PHCA})$ is conservative over full bounded arithmetic $\Sigma_{\infty}^{b}-\mathrm{NIA}$ for $\forall \exists \Sigma_{\infty}^{b}$ sentences. This will yield, in particular, that the $\Sigma_{1}^{b}$ definable functions of $\mathbb{A}(\mathrm{PTCA})$ are the polytime functions, and (ii) the $\Sigma_{\infty}^{b}$ definable functions of $\mathbb{A}(\mathrm{PHCA})$ are the functions in the polynomial time hierarchy.

The plan of this paper is as follows. In Sect. 2 we give a detailed introduction to Ferreira's language and systems of polynomial time and polynomial hierarchy computable arithmetic. We further introduce (and analyze) two well-known reflection principles in the context of bounded arithmetic which will later be used in our analysis of weak set theories, namely sharp $\Sigma$ reflection and bounded collection. In Sect. 3 we define the two admissible closures $\mathbb{A}(P T C A)$ and $\mathbb{A}(P H C A)$ stipulated by the set existence axioms mentioned above. In Sect. 4 we show by a straightforward embedding argument that $\mathbb{A}(\mathrm{PTCA})$ is contained in PTCA plus sharp $\Sigma$ reflection. In Sect. 5 it is established via a two-step model-theoretic argument that $\mathbb{A}(\mathrm{PHCA})$ is conservative over PHCA augmented by the schema of bounded collection. In an intermediate step we will consider a second order arithmetical theory with bounded comprehension and a finite axiom of choice. The paper ends in Sect. 6 with conclusions and a short discussion of related work in Feferman's explicit mathematics, Sazonov's bounded set theory, and Sato's weak weak set theories. 
The results of this paper were first presented at the workshop Proof, Computation, Complexity PPC '07, 13-14 April 2007, Swansea, Wales.

\section{Polynomial time computable arithmetic}

The theory PTCA of polynomial time computable arithmetic over binary strings was introduced by Ferreira [8,9]. It provides an approach to weak arithmetic which is similar in spirit to Buss' Bounded Arithmetic (cf. Buss [1]), but instead of natural numbers being grounded on a language of binary words. PTCA can be viewed as a polynomial time analogue of Skolem's system of primitive recursive arithmetic PRA. The theory PTCA is formulated in the first order language $L_{p}$, which is based on the elementary language $\mathrm{L}$. The latter language includes variables $a, b, c, u, v, w, x, y, z, \ldots$, the constants $\varepsilon, 0,1$ (empty word, zero, one), the binary function symbols $*$ and $\times$ (word concatenation and word multiplication) and the binary relation symbol $\sqsubseteq$ (initial subword relation). Here $u \times v$ denotes the word $u$ concatenated with itself length of $v$ times; moreover, $u \sqsubseteq v$ holds iff $v=u * w$ for some word $w$. We will often write $u v$ for $u * v$.

The language $L$ is characterized by the following fourteen basic axioms:

$$
\begin{array}{ll}
u \varepsilon=u & u \times \varepsilon=\varepsilon \\
u(v 0)=(u v) 0 & u \times(v 0)=(u \times v) u \\
u(v 1)=(u v) 1 & u \times(v 1)=(u \times v) u \\
u \sqsubseteq \varepsilon \leftrightarrow u=\varepsilon & u 0=v 0 \rightarrow u=v \\
u \sqsubseteq v 0 \leftrightarrow u \sqsubseteq v \vee u=v 0 & u 1=v 1 \rightarrow u=v \\
u \sqsubseteq v 1 \leftrightarrow u \sqsubseteq v \vee u=v 1 & u 0 \neq v 1 u 0 \neq \varepsilon \quad u 1 \neq \varepsilon
\end{array}
$$

The language $L_{p}$ is obtained from $L$ by adding a function symbol for each description of a polynomial time computable function, where the terms of $L$ act as bounding terms, similar to Cobham's characterization of the polynomial time computable functions (cf. [4]). More precisely, the polytime functions can be generated inductively with the schemata of composition and bounded iteration from a set of initial functions $E, P_{i}^{n}(1 \leq i \leq n), C_{0}, C_{1}, Q$. The initial functions are defined by

1. $E(u)=\varepsilon$

2. $P_{i}^{n}\left(u_{1}, \ldots, u_{n}\right)=u_{i}$;

3. $C_{0}(u)=u 0$;

4. $C_{1}(u)=u 1$;

5. $Q(u, v)=1$ if $u \sqsubseteq v$ and 0 , otherwise.

$f$ is defined by composition from $g, h_{1}, \ldots h_{k}$ if $f$ satisfies

6. $f\left(u_{1}, \ldots, u_{n}\right)=g\left(h_{1}\left(u_{1}, \ldots, u_{n}\right), \ldots, h_{k}\left(u_{1}, \ldots, u_{n}\right)\right)$.

$f$ is defined by bounded iteration from $g, h_{0}, h_{1}$ with bound $t$ if

$7.1 f\left(u_{1}, \ldots, u_{n}, \varepsilon\right)=g\left(u_{1}, \ldots, u_{n}\right)$;

$7.2 f\left(u_{1}, \ldots, u_{n}, v i\right)=\left.h_{i}\left(u_{1}, \ldots, u_{n}, v, f\left(u_{1}, \ldots, u_{n}, v\right)\right)\right|_{t\left(u_{1}, \ldots, u_{n}, v\right)}$, 
where $i=0,1, t$ is an $\mathrm{L}$ term ${ }^{1}$ and $\left.u\right|_{w}$ denotes the truncation of $u$ to the length of $w$. Observe that $\mid$ is definable by a quantifier-free formula of $L$, cf. [8,9].

The terms $(r, s, t, \ldots)$ of $\mathrm{L}_{p}$ are defined as usual. Atoms have the form $t=s$ or $t \sqsubseteq s$. Literals are atoms or negated atoms. The formulas $(A, B, C, \ldots)$ of $\mathrm{L}_{p}$ are generated from the literals by means of $\wedge, \vee, \forall$, and $\exists$. We will use the following abbreviations:

$$
s \sqsubseteq * t:=\exists x(x \sqsubseteq t \wedge x s \sqsubseteq t), \quad s \leq t:=1 \times s \sqsubseteq 1 \times t .
$$

Hence, $s \sqsubseteq^{*} t$ holds if $s$ is a subword of $t$ and $s \leq t$ means that the length $|s|$ of $s$ is less than or equal than the length of $t$.

Suppose that the variable $x$ does not appear in the term $t$ and $R=\sqsubseteq^{*}, \sqsubseteq, \leq$. Then we use the shorthand notations

$$
(\forall x R t) A:=\forall x(x R t \rightarrow A) \text { and }(\exists x R t) A:=\exists x(x R t \wedge A)
$$

The quantifiers $\left(\forall x \sqsubseteq^{*} t\right)$ as well as $\left(\exists x \sqsubseteq^{*} t\right)$ are called subword quantifiers or sharply bounded quantifiers; the quantifiers $(\forall x \leq t)$ and $(\exists x \leq t)$ are called bounded quantifiers.

The class of $\Delta_{0}^{b}$ formulas is the smallest class of formulas of $\mathrm{L}_{p}$ that is generated from literals by means of conjunction, disjunction and sharply bounded quantification. An $\mathrm{L}_{p}$ formula is called $\Sigma_{1}^{b}$ if it is of the form $(\exists x \leq t) A(x)$ with $A$ a $\Delta_{0}^{b}$ formula. Moreover, a formula is called bounded or $\Sigma_{\infty}^{b}$ if all its quantifiers are bounded in the sense of $\leq$.

Ferreira's system PTCA of polynomial time computable arithmetic is now defined to be the first order theory based on classical logic with equality, and comprising defining axioms for the function and relation symbols of the language $L_{p}$. In addition, PTCA includes the schema of notation induction on binary words for quantifier free formulas, i.e. it includes the axiom

$$
A(\varepsilon) \wedge \forall x(A(x) \rightarrow A(x 0) \wedge A(x 1)) \rightarrow \forall x A(x)
$$

for each quantifier-free formula $A(u)$ of $\mathrm{L}_{p}$. It is well-known that PTCA proves notation induction for $\Delta_{0}^{b}$ formulas, because each $\Delta_{0}^{b}$ formula is provably equivalent in PTCA to a quantifier-free formula (cf. [8,9,3]).

A well-studied expansion of PTCA is the theory $\mathrm{PTCA}^{+}$(cf. [9]) which extends PTCA by the schema of notation induction for $\Sigma_{1}^{b}$ formulas of $L_{p}$. It is well-known that $\mathrm{PTCA}^{+}$is a conservative extension of PTCA for $\forall \exists \Sigma_{1}^{b}$ statements and, hence, its provably total functions are the polytime functions. Moreover, in $\mathrm{PTCA}^{+}$one can dispense with the functions symbols for polytime functions as these can be $\Sigma_{1}^{b}$ defined using $\Sigma_{1}^{b}$ induction in the restricted language L (cf. [9]).

We will also be interested in the extension of $\mathrm{PTCA}^{+}$where notation induction is permitted for all bounded or $\Sigma_{\infty}^{b}$ formulas of $L_{p}$. This system is denoted by $\Sigma_{\infty}^{b}-\mathrm{NIA}$ in [10]. The $\Sigma_{\infty}^{b}$ definable functions of this theory are exactly the functions in the Meyer

1 Note that we interpret $\lambda x_{1} \ldots x_{n+1} . t\left(x_{1}, \ldots, x_{n+1}\right)$ in the standard model. 
Stockmeyer polynomial time hierarchy. We will use the name PHCA (polynomial hierarchy computable arithmetic) instead of $\Sigma_{\infty}^{b}$-NIA in this paper.

Later we will also be interested in suitable extensions of PTCA and PHCA by reflection principles. Thereby, PTCA $\sharp$ is PTCA strengthened by sharp $\Sigma$ reflection, and $\mathrm{PHCA}^{\natural}$ is PHCA plus bounded collection. Sharp $\Sigma$ reflection states that

$$
(\Sigma \text {-sRef }) \quad\left(\forall x \sqsubseteq^{*} b\right) \exists y A(x, y) \rightarrow \exists z\left(\forall x \sqsubseteq^{*} b\right)\left(\exists y \sqsubseteq^{*} z\right) A(x, y),
$$

for each $\Delta_{0}^{b}$ formula $A(u, v)$ of $\mathrm{L}_{p}$, and bounded collection claims for each $\Sigma_{\infty}^{b}$ formula $A(u, v)$ of $\mathrm{L}_{p}$

$$
(\Sigma \text {-bColl }) \quad(\forall x \leq b) \exists y A(x, y) \rightarrow \exists z(\forall x \leq b)(\exists y \leq z) A(x, y)
$$

The following lemma will be crucial in the upper bound computations of $\mathbb{A}(\mathrm{PTCA})$ and $\mathbb{A}(\mathrm{PHCA})$.

Lemma $1 \mathrm{PTCA}^{\sharp}$ proves the same $\forall \exists \Delta_{0}^{b}$ sentences as $\mathrm{PTCA}$, and $\mathrm{PHCA}^{\natural}$ proves the same $\forall \exists \Sigma_{\infty}^{b}$ sentences as $\mathrm{PHCA}$.

Proof This is a consequence of a stronger result by Cantini [3], cf. also Buss [2] and Ferreira [11]. However, we provide a direct model theoretic argument that is similar in spirit to the proof of our main result (cf. Lemma 8). The contraposition of the nontrivial direction of the lemma is shown by proving that if $C:=\forall x \exists y A(x, y)$ is a $\forall \exists \Delta_{0}^{b}$ $\left[\forall \exists \Sigma_{\infty}^{b}\right]$ sentence of $\mathrm{L}_{p}$ and $\neg C$ is consistent with PTCA [PHCA], then $\neg C$ is also consistent with $\mathrm{PTCA}^{\sharp}\left[\mathrm{PHCA}^{\natural}\right]$. We just consider the case PTCA. The argument for $\mathrm{PHCA}$ runs analogously but is simpler.

Below, $\left(f_{i}: i \in \mathbb{N}\right)$ is an enumeration of the unary polytime function symbols of $\mathrm{L}_{p}$. Further, if $f\left(w_{1}, \ldots, w_{n}\right)$ is an $n$-ary polytime function on words, then $g(w):=\Sigma_{\vec{y} \sqsubset^{*} w} f(\vec{y})$ denotes a fixed polytime function with the property that $\left(\forall \vec{v} \sqsubseteq^{*} w\right)\left(f(\vec{v}) \sqsubseteq^{*} g(w)\right)$. It is a routine matter to check that such a polytime function indeed exists.

Assume that $A(u, v)$ is a $\Delta_{0}^{b}$ formula of $\mathrm{L}_{p}$ and that $\mathcal{W}_{0}=\left(\mathrm{W}_{0}, \ldots\right) \models \mathrm{PTCA}$ so that $\mathcal{W}_{0} \models \exists x \forall y \neg A(x, y)$. Hence, $\mathcal{W}_{0} \models \forall y \neg A(\mathbf{w}, y)$ for some $\mathbf{w} \in \mathbf{W}_{0}^{2}$.

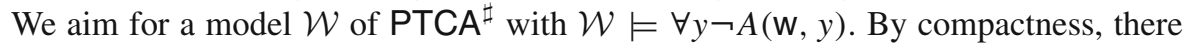
is a model $\mathcal{W}^{\prime}$ of PTCA that satisfies $\forall y \neg A(\mathrm{~W}, y)$ and contains a word $\mathrm{C}$ so that for each $n \in \mathbb{N}, f_{0}^{\mathcal{W}^{\prime}}(\mathrm{W}) * \cdots * f_{n}^{\mathcal{W}^{\prime}}(\mathrm{w}) \sqsubseteq \mathrm{c}$. Then $\mathcal{W}, \mathcal{W}^{\prime}$ restricted to the domain $\mathrm{W}:=\left\{\mathrm{v}:(\exists n \in \mathbb{N})\left(\left.\mathrm{v} \sqsubseteq^{*} \mathrm{C}\right|_{f_{n}^{\mathcal{W}^{\prime}}(\mathrm{w})}\right\}\right.$, is the desired model of $\mathrm{PTCA}^{\sharp}:$ By definition, $\mathrm{v}^{\prime} \sqsubseteq^{*} \mathrm{v}$ and $\mathrm{v} \in \mathrm{W}$ imply $\mathrm{v}^{\prime} \in \mathrm{W}$. If $\overrightarrow{\mathrm{v}} \in \mathrm{W}$, then there are $i$ and $j$ so that $\overrightarrow{\mathrm{v}} \sqsubseteq *$

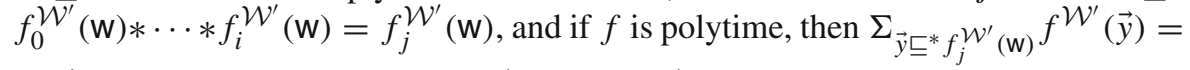
$f_{n}^{\mathcal{W}^{\prime}}(\mathrm{w})$ for some $n$. Therefore, $\left.f^{\mathcal{W}^{\prime}}(\overrightarrow{\mathrm{v}}) \sqsubseteq^{*} f_{n}^{\mathcal{W}^{\prime}}(\mathrm{w}) \sqsubseteq^{*} \mathrm{c}\right|_{f_{0}^{\mathcal{W}}} \mathcal{W}^{\prime}(\mathrm{w}) * \cdots * f_{n}^{\mathcal{W} \mathcal{W}^{\prime}(\mathrm{w})}$. Hence, $\mathrm{W}$ is closed under polytime functions. It remains to check that $\mathcal{W}$ satisfies ( $\Sigma$-sRef). So suppose that $\mathcal{W} \models\left(\forall x \sqsubseteq^{*} \mathrm{~b}\right) \exists y B(x, y)$ for some $\Delta_{0}^{b}$ formula $B(u, v)$ of $\mathrm{L}_{p}$. Let

\footnotetext{
${ }^{2}$ Sans-serif letters denote elements of the domain of the model of discourse: for instance, $\mathcal{W}_{0} \models A(\mathrm{w})$ is short for $\mathcal{W}_{[u=\mathrm{w}]} \models A(u)$.
} 


$$
\begin{aligned}
\emptyset \neq \mathcal{X} & :=\left\{\mathrm{z} \sqsubseteq \mathrm{c}: \mathcal{W}^{\prime} \models\left(\forall x \sqsubseteq^{*} \mathrm{~b}\right)\left(\exists y \sqsubseteq^{*} \mathrm{z}\right) B(x, y)\right\} \\
& \supseteq\{\mathrm{v} \sqsubseteq \mathrm{c}: \mathrm{v} \notin \mathrm{W}\}=: \mathcal{Y} .
\end{aligned}
$$

Since $\mathrm{z}_{0}:=\min _{\sqsubseteq}(\mathcal{X})$ exists by $\Delta_{0}^{b}$ induction and $\mathrm{z}_{0} \in \mathcal{Y}$ is impossible as $\mathcal{Y}$ has no $\sqsubseteq$-minimal element, $\mathrm{z}_{0} \in \mathrm{W}$, and $\mathcal{W} \models\left(\forall x \sqsubseteq^{*} \mathrm{~b}\right)\left(\exists y \sqsubseteq^{*} \mathrm{z}_{0}\right) B(x, y)$. This concludes our proof.

\section{Two admissible closures}

In the following we define two natural admissible closures $\mathbb{A}(P T C A)$ and $\mathbb{A}(P H C A)$ of PTCA and PHCA, respectively. Later we will show that these closures do not raise the proof-theoretic strength of PTCA and PHCA.

$\mathbb{A}(\mathrm{PTCA})$ and $\mathbb{A}(\mathrm{PHCA})$ are formulated in the extension $\mathcal{L}^{*}=L_{p}(\in, \mathrm{W}, \mathrm{S})$ of $\mathrm{L}_{p}$ by the membership relation symbol $\in$ and the unary relation symbols $W$ and $S$ for the class of binary words and sets, respectively.

The terms $(r, s, t, \ldots)$ of $\mathcal{L}^{*}$ are the terms of $\mathrm{L}_{p}$. The formulas $(A, B, C, \ldots)$ of $\mathcal{L}^{*}$ as well as the $\Delta_{0}$ formulas of $\mathcal{L}^{*}$ are defined as usual; i.e., an $\mathcal{L}^{*}$ formula is $\Delta_{0}$ if it is built from positive or negative literals by means of conjunction, disjunction and the bounded quantifiers $(\forall x \in s)$ as well as $(\exists x \in s)$. The notation $\vec{s}$ is shorthand for a finite string $s_{1}, \ldots, s_{n}$ whose length will be specified by the context. Equality between objects is not represented by a primitive symbol but defined by

$$
(s=\mathrm{W}, \mathrm{S} t):=\left\{\begin{array}{l}
(\mathrm{W}(s) \wedge \mathrm{W}(t) \wedge(s=t) \vee \\
(\mathrm{S}(s) \wedge \mathrm{S}(t) \wedge(\forall x \in s)(x \in t) \wedge(\forall x \in t)(x \in s))
\end{array}\right.
$$

By slight abuse of notation, we will often write $s=t$ instead of $s=\mathrm{w}, \mathrm{S} t$ when working in the language $\mathcal{L}^{*}$. Moreover, we use the following shorthand notation

$$
s=\{x: A(x)\}:=(\forall x \in s) A(x) \wedge \forall x(A(x) \rightarrow x \in s)
$$

For an $\mathrm{L}_{p}$ formula $A$ we write $A^{\mathrm{W}}$ for its relativization to the class $\mathrm{W}$.

In the sequel we write $t[\vec{s} / \vec{u}]$ and $A[\vec{s} / \vec{u}]$ for the substitution of the terms $\vec{s}$ for the variables $\vec{u}$ in $t$ and $A$, respectively. If the variables $\vec{u}$ are clear from the context, we sometimes write $t(\vec{s})$ and $A(\vec{s})$ instead of $t[\vec{s} / \vec{u}]$ and $A[\vec{s} / \vec{u}]$. As usual, we let $\mathrm{FV}(t)$ and $\mathrm{FV}(A)$ stand for the set of free variables of $t$ and $A$, respectively.

Let us now first introduce the admissible closure $\mathbb{A}(\mathrm{PTCA})$. Its logical axioms comprise the usual axioms of classical first order logic with equality. The non-logical axioms of $\mathbb{A}(\mathrm{PTCA})$ can be divided into the following groups.

I. Ontological axioms, part A. We have for all function symbols $h$ and relation symbols $R$ of the language $\mathrm{L}_{p}$ :

$$
\mathrm{W}(a) \leftrightarrow \neg \mathrm{S}(a), \quad \mathrm{W}(\vec{b}) \rightarrow \mathrm{W}(h(\vec{b})), \quad R(\vec{b}) \rightarrow \mathrm{W}(\vec{b}), \quad a \in b \rightarrow \mathrm{S}(b) .
$$


II. Ontological axioms, part B. Here we include the crucial axiom (W.0) which claims that the collection of all subwords of a binary word forms a set:

$$
\text { (W.0) } \mathbf{W}(a) \rightarrow \exists x\left(\mathbf{S}(x) \wedge x=\left\{y: \mathbf{W}(y) \wedge y \sqsubseteq^{*} a\right\}\right) .
$$

III. Axioms about W. We have for all axioms $A(\vec{u})$ of PTCA except induction, with just the displayed variables free:

$$
(\mathrm{W} \text { axioms }) \mathrm{W}(\vec{a}) \rightarrow A^{\mathrm{W}}(\vec{a}) .
$$

IV. Kripke-Platek axioms. We have for all $\Delta_{0}$ formulas $A(u)$ and $B(u, v)$ of the language $\mathcal{L}^{*}$ :

(Pair) $\exists x(a \in x \wedge b \in x)$,

(Union) $\exists x(\forall y \in a)(\forall z \in y)(z \in x)$,

$\left(\Delta_{0}\right.$-Sep) $\exists x(\mathrm{~S}(x) \wedge x=\{y \in a: A(y)\})$,

$\left(\Delta_{0}\right.$-Coll) $\quad(\forall x \in a) \exists y B(x, y) \rightarrow \exists z(\forall x \in a)(\exists y \in z) B(x, y)$.

V. Foundation. Here we include the usual regularity axiom:

$$
\text { (Fund) } \mathrm{S}(a) \wedge a \neq \emptyset \rightarrow(\exists x \in a)(\forall y \in x)(y \notin a) .
$$

VI. $\Delta_{0}$ induction on W. We have $\Delta_{0}$ notation induction on the class of binary words W, i.e. for each $\Delta_{0}$ formula $A(u)$ of $\mathcal{L}^{*}$ :

$$
\left(\Delta_{0}-\mathrm{I}_{\mathrm{W}}\right) \quad A(\varepsilon) \wedge(\forall x \in \mathrm{W})[A(x) \rightarrow A(x 0) \wedge A(x 1)] \rightarrow(\forall x \in \mathrm{W}) A(x) .
$$

This concludes our description of $\mathbb{A}(P T C A)$. Whereas the crucial set existence axiom with respect to the class $W$ in $\mathbb{A}(P T C A)$ claims the existence of the set of all subwords of a given word $a$, in the stronger closure $\mathbb{A}(\mathrm{PHCA})$ it is claimed that for each word $a$ we have the set of all words $b$ whose length is less than or equal to the length of $a$. More precisely, $\mathbb{A}(\mathrm{PHCA})$ is obtained from $\mathbb{A}(\mathrm{PTCA})$ by replacing (W.0) by the stronger axiom (W.1):

$$
\text { (W.1) } \mathbf{W}(a) \rightarrow \exists x(\mathrm{~S}(x) \wedge x=\{y: \mathrm{W}(y) \wedge y \leq a\}) .
$$

Observe that $\mathbb{A}(\mathrm{PHCA})$ proves the weaker axiom (W.0). We further let $\mathbb{A}\left(\mathrm{PTCA}^{\sharp}\right)$ be defined as $\mathbb{A}(P T C A)$, but with PTCA replaced by PTCA $A^{\sharp}$ in the definition of the axioms in group III. $\mathbb{A}\left(\mathrm{PHCA}^{\natural}\right)$ is defined accordingly.

Clearly, PTCA is contained in $A(P T C A)$, since notation induction on $W$ for quantifier-free formulas of $L_{p}$ follows from $\left(\Delta_{0}-I_{W}\right)$. In order to see that the stronger system PHCA is contained in the stronger admissible closure $\mathbb{A}(\mathrm{PHCA})$ we need a little bit of elaboration.

Recall from Sect. 2 that by PHCA we denote the system PTCA with induction extended to all $\Sigma_{\infty}^{b}$ formulas, i.e., formulas all of whose quantifiers are bounded with respect to the relation $\leq$. In order to verify induction for all bounded formulas, let 
us recall that in the language of PTCA, each term $t$ of $\mathrm{L}_{p}$ with $\mathrm{FV}(t)=\{\vec{u}\}$ can be majorized by a term $t^{\prime}$ of $\mathrm{L}$, i.e.

$$
\mathrm{PTCA} \vdash \forall \vec{x}\left(t(\vec{x}) \leq t^{\prime}(\vec{x})\right)
$$

Moreover, terms of $L$ are provably $\leq$ monotone in PTCA. These two facts imply that terms of $\mathrm{L}_{p}$ are provably majorized by $\mathrm{a} \leq$ monotone term of $\mathrm{L}$. The above observations readily entail that for each $\Sigma_{\infty}^{b}$ formula $A$ with $\mathrm{FV}(A)=\{\vec{u}\}$, there are terms $t_{1}, \ldots, t_{n}$ with $\mathrm{FV}\left(t_{i}\right) \subseteq\{\vec{u}\}$ and a quantifier-free formula $B$ with $\mathrm{FV}(B) \subseteq\left\{\vec{u}, v_{1}, \ldots, v_{n}\right\}$ so that (provably in PTCA) $A$ is equivalent to

$$
\left(\mathcal{Q}_{1} y_{1} \leq t_{1}\right)\left(\mathcal{Q}_{2} y_{2} \leq t_{2}\right) \ldots\left(\mathcal{Q}_{n} y_{n} \leq t_{n}\right) B\left(\vec{u}, y_{1}, y_{2}, \ldots, y_{n}\right)
$$

where $\mathcal{Q}_{i} \in\{\exists, \forall\}$. Hence, we can define $A$ by a $\Delta_{0}$ formula in $\mathcal{L}^{*}$ by using $(\mathrm{W} .1)$ in order to define the sets

$$
a_{i}:=\left\{z \in \mathrm{W}: z \leq t_{i}\right\} \quad(1 \leq i \leq n)
$$

and then consider the $\Delta_{0}$ formula

$$
\left(\mathcal{Q}_{1} y_{1} \in a_{1}\right)\left(\mathcal{Q}_{2} y_{2} \in a_{2}\right) \ldots\left(\mathcal{Q}_{n} y_{n} \in a_{n}\right) B\left(\vec{u}, y_{1}, y_{2}, \ldots, y_{n}\right)
$$

Given these preparatory steps, induction for $\Sigma_{\infty}^{b}$ formulas in PHCA follows from $\left(\Delta_{0}-I_{W}\right)$ in $\mathbb{A}(P H C A)$. To summarize, we can state the following embedding results:

Lemma 2 For each $\mathrm{L}_{p}$ formula $A(\vec{u})$ with just the displayed variables free we have:

1. $\mathrm{PTCA} \vdash A(\vec{u}) \Longrightarrow \mathbb{A}(\mathrm{PTCA}) \vdash \vec{u} \in \mathrm{W} \rightarrow A^{\mathrm{W}}(\vec{u})$.

2. $\mathrm{PHCA} \vdash A(\vec{u}) \Longrightarrow \mathbb{A}(\mathrm{PHCA}) \vdash \vec{u} \in \mathrm{W} \rightarrow A^{\mathrm{W}}(\vec{u})$.

\section{Embedding A (PTCA) into PTCA}

The idea is to embed $\mathbb{A}\left(\right.$ PTCA) into PTCA $^{\sharp}$ by representing sets as binary words. This is possible because the initial sets $\left\{w: w \sqsubseteq{ }^{*} a\right\}$ of $\mathbb{A}(\mathrm{PTCA})$ have only about $|a|^{2}$ many elements and can be represented by a single word.

First, we introduce a couple of polytime functions and relations: To code finite sequences of words, we let $\varepsilon^{*}:=\varepsilon$ and $(w i)^{*}:=w^{*} 1 i$ for $i \in\{0,1\}$, and then $\left\langle w_{0}, \ldots, w_{n}\right\rangle_{\text {seq }}:=00 w_{0}^{*} 00 w_{1}^{*} 00 \cdots 00 w_{n}^{*}$. The predicate $\operatorname{seq}(u)$ distinguishes words coding sequences, Ih is a function so that $\operatorname{lh}\left(\left\langle w_{0}, \ldots, w_{n}\right\rangle_{\text {seq }}\right)$ returns a string of $n$ zeros and $\pi$ a function so that for each word $b$ with length $i, \pi\left(\left\langle w_{0}, \ldots, w_{n}\right\rangle_{\text {seq }}, b\right)=w_{i}$. Further, we agree that $\operatorname{word}(w)$ iff $w \in 10 \mathrm{~W}$, i.e. if $w$ is of the form $10 w^{\prime}$. The unary relation $\operatorname{set}(w)$ distinguishes words which code sets: $11 \in$ set is a code of the empty set, and if $w_{0}<$ lex $\ldots<$ lex $w_{n}{ }^{3}$ are elements of

\footnotetext{
${ }^{3}$ Here $<_{\text {lex }}$ denotes the ordering according to which words are ordered by their length and words of the same length are ordered lexicographically.
} 
set $\cup$ word, then $w=\left\langle w_{0}, \ldots, w_{n}\right\rangle_{\text {seq }} \in$ set codes the set containing the sets or words coded by $w_{0}, \ldots, w_{n}$. Finally, $\operatorname{obj}(w):=\operatorname{word}(w) \vee \operatorname{set}(w), \operatorname{el}(a, b)$ iff $\operatorname{set}(b)$ and $\operatorname{obj}(a)$ and $\pi(b, i)=a$ for some $i<\ln (b), \operatorname{con}\left(\left\langle w_{0}, \ldots, w_{n}\right\rangle_{\text {seq }}\right):=w_{0} w_{1} \cdots w_{n}$, and tail is such that for all words $w$, tail $(10 w):=w$. Note that el $(a, b)$ implies $a \sqsubseteq * \operatorname{con}(b)$.

Next, we assign to each term $t$ of $\mathcal{L}^{*}$ a term $t^{\circ}$ of $\mathrm{L}_{p}$, and to each formula $A$ of $\mathcal{L}^{*}$ a formula $A^{\circ}$ of $\mathrm{L}_{p}$. For variables, $u_{i}^{\circ}:=u_{i}, c^{\circ}:=10 c$ if $c$ is a word constant (there are no set constants!) and $\left(f\left(t_{1} \ldots, t_{n}\right)\right)^{\circ}:=10 f\left(\right.$ tail $\left(t_{1}^{\circ}\right), \ldots$, tail $\left.\left(t_{n}^{\circ}\right)\right)$. If $R$ is a relation symbol of $\mathrm{L}_{p}$, then $(R(\vec{t}))^{\circ}:=R\left(\right.$ tail $\left.\left(\vec{t}^{\circ}\right)\right) . \mathrm{W}(t)$ translates to word $\left(t^{\circ}\right), \mathrm{S}(t)$ to $\operatorname{set}\left(t^{\circ}\right)$ and $s \in t$ to $\mathrm{el}\left(s^{\circ}, t^{\circ}\right)$. This translations canonically extends to all formulas of $\mathcal{L}^{*}$, applying $(\mathcal{Q} x A(x))^{\circ}:=(\mathcal{Q} x \in$ obj $) A^{\circ}(x)$ for unbounded quantifiers.

Lemma 3 For each $\mathcal{L}^{*}$ formula $A(\vec{u})$ with just the displayed variables free,

$$
\mathbb{A}(\mathrm{PTCA}) \vdash A(\vec{u}) \quad \Longrightarrow \quad \mathrm{PTCA}^{\sharp} \vdash \operatorname{obj}(\vec{u}) \rightarrow A^{\circ}(\vec{u}) \text {. }
$$

Proof It is easily checked that if $A$ is a formula of $\mathrm{L}_{p}$ with $\mathrm{FV}(A)=\{\vec{u}\}$, then, provably in $\mathrm{PTCA}^{\sharp}, \forall \vec{x}\left(\mathrm{~W}(\vec{x}) \rightarrow A^{\mathrm{W}}\right)^{\circ}$ is equivalent to $\forall \vec{x} A$. Extensionality follows by our coding of sets, i.e. PTCA proves

$$
\operatorname{set}(a) \wedge \operatorname{set}(b) \wedge \forall x[\operatorname{el}(x, a) \leftrightarrow \operatorname{el}(x, b)] \rightarrow a=b .
$$

There are codes for sets of the form $\left\{w: w \sqsubseteq{ }^{*} b\right\}$, and 11 is the unique code of $\emptyset$. If $a, b$ code objects and $a<_{\operatorname{lex}} b$, then $\mathrm{el}\left(x,\langle a, b\rangle_{\mathrm{seq}}\right)$ implies that $x=a \vee x=b$. If $a=\left\langle b_{0} \ldots b_{n}\right\rangle_{\text {seq }}$ codes a set $x$, then a code of $\bigcup x$ is computed from $a$ in polynomial time: Just arrange the words $c=\pi\left(b_{i}, j\right)\left(0 \leq j<\ln \left(b_{i}\right)\right)$ occurring in those $b_{i}$ 's that code sequences in a $<_{\text {lex }}$-ascending sequence and remove doublets.

Since el $(a, b)$ entails $a \sqsubseteq{ }^{*} \operatorname{con}(b)$, the translation of a $\Delta_{0}$ formula $A$ of $\mathcal{L}^{*}$ is equivalent to a $\Delta_{0}^{b}$ formula of $\mathrm{L}_{p}$. This readily implies $\Delta_{0}$ separation. And if $A(u)$ is $\Delta_{0}$, then the translation of $(\forall x \in a) \exists y A(x, y)$ is equivalent to

$$
\left(\forall x \sqsubseteq{ }^{*} \operatorname{con}(a)\right) \exists y\left[\operatorname{el}(x, a) \rightarrow \operatorname{obj}(y) \wedge A^{\circ}(x, y)\right] .
$$

Using sharp $\Sigma$ reflection one obtains a code $b \in$ set so that

$$
\left(\forall x \sqsubseteq^{*} \operatorname{con}(a)\right)\left(\exists y \sqsubseteq^{*} b\right)\left[\mathrm{el}(x, a) \rightarrow \mathrm{el}(y, b) \wedge A^{\circ}(x, y)\right],
$$

which validates the translation of $\exists b(\forall x \in a)(\exists y \in b) A(x, y)$.

Lemma 1, Lemma 2, and Lemma 3 now yield the following theorem.

Theorem 4 PTCA and $\mathbb{A}(\mathrm{PTCA})$ prove the same $\forall \exists \Delta_{0}^{b}$ sentences.

\section{Conservativity of $\mathbb{A}\left(\right.$ PHCA) over PHCA ${ }^{\natural}$}

Our strategy is to establish that $\mathrm{PHCA}^{\natural}$ and $\mathbb{A}\left(\mathrm{PHCA}^{\natural}\right)$ prove the same $\mathrm{L}_{p}$ formulas by showing that any model $\mathcal{W}_{0}=\left(\mathrm{W}_{0}, \complement^{\mathcal{W}_{0}}, \ldots\right)$ of $\mathrm{PHCA}^{\natural}$ can be transformed into 
a model $(\mathcal{W}, \mathcal{A})$ of $\mathbb{A}\left(\mathrm{PHCA}^{\natural}\right)$ that still satisfies the same $\mathrm{L}_{p}$ sentences. The predicate $\mathrm{W}(u)$ of $\mathcal{L}^{*}$ is interpreted as the universe of $\mathcal{W}$, and $\mathrm{S}(u)$ as $u \in \mathcal{A}$, a suitable collection of sets with urelements from $\mathrm{W}$. The $\in$ relation is the restriction of the standard $\in$ relation to $\mathrm{W} \cup \mathcal{A} \times \mathcal{A}$. By Lemma $1, \mathrm{PHCA}$ and $\mathbb{A}(\mathrm{PHCA})$ prove the same $\forall \exists \Sigma_{\infty}^{b}$ sentences.

Our model construction depends on a coding of sets in the cumulative hierarchy above the domain $\mathrm{W}$ of some model $\mathcal{W}$ of $\mathrm{PHCA}^{\natural}$ as subsets of $\mathrm{W}$. We define $\langle u, v\rangle:=00 u 00 v^{*}$ and let pair denote the polytime relation that contains $w:=\langle u, v\rangle$ iff $u=11 \vee u \in 10 \mathrm{~W} \vee u \in$ pair. Note that $\mathrm{W} \in$ pair $^{\mathcal{W}}$ starts with an even number of zeros. By Rep we denote the subsets $\mathcal{X}$ of $\mathrm{W}$ that are used to represent sets in the cumulative hierarchy above $\mathrm{W}$.

$$
\mathcal{X} \in \operatorname{Rep}^{\mathcal{W}}: \Leftrightarrow(\forall x \in \mathcal{X})\left(x=11 \vee x \in 10 \mathrm{~W} \vee x \in \text { pair }^{\mathcal{W}}\right)
$$

Henceforth we mostly drop the superscript $\mathcal{W}$, but bear in mind that our definitions are relative to some model $\mathcal{W}$ of $\mathrm{PHCA}^{\natural}$. By the definition of pair, $\mathcal{X} \in$ Rep implies that $(\mathcal{X})_{\mathrm{w}}:=\{\mathrm{v}:\langle\mathrm{v}, \mathrm{w}\rangle \in \mathcal{X}\} \in \operatorname{Rep}$.

We say that $\mathrm{w}$ is a bound for the width of $\mathcal{X}$, or synonymously, that the width of $\mathcal{X}$ is bounded by $\mathrm{w}$, if $(\forall x \in \mathcal{X})(x \leq \mathrm{w})$. Accordingly, w is a bound for the depth of $\mathcal{X}$, if $(\forall x \in \mathcal{X})(00 \times \mathrm{w} 0 \nsubseteq x)$. If $<_{\text {lex }}$-least such bounds exist, they are referred to as the width and the depth of $\mathcal{X}$, respectively. Subsequently, we abbreviate $(\forall x \in \mathcal{X})(x \leq \mathrm{w})$ by wth $(\mathcal{X}) \leq \mathrm{w}$, and $(\forall x \in \mathcal{X})(00 \times \mathrm{w} 0 \nsubseteq x)$ by $\mathrm{dth}(\mathcal{X}) \leq \mathrm{w}$. Note however, that in general $\mathcal{X}$ does not have a depth or a width. Further, $\operatorname{dth}(\mathcal{X}, \mathcal{Y}) \leq \mathrm{w}$ states that the depths of $\mathcal{X}$ and $\mathcal{Y}$ are bounded by $\mathrm{w}$, and $w$ th $(\mathcal{X}) \leq \mathrm{W}$ expresses that the width of $\mathcal{X}$ is bounded by some $\mathrm{w} \in \mathrm{W}$. Moreover, $\mathcal{X} \in \operatorname{Rep} \mid \mathrm{w}$ iff $\mathcal{X} \in \operatorname{Rep}$ and $\operatorname{dth}(\mathcal{X}) \leq \mathrm{w}$, and $\mathcal{X} \in \operatorname{Rep}\left\lceil\mathbb{W}^{4}\right.$ iff $\mathcal{X} \in \operatorname{Rep}$ and $\operatorname{dth}(\mathcal{X}) \leq \mathbb{W}$, and $\mathcal{X} \in \operatorname{Rep}^{*}$ iff $\mathcal{X} \in \operatorname{Rep}\lceil\mathbb{W}$ and $w \operatorname{th}(\mathcal{X}) \leq \mathrm{W}$. For $\mathcal{X} \in \operatorname{Rep} \uparrow \mathbb{W}$, we can define the extension of the set coded by $\mathcal{X}$,

$$
\begin{aligned}
\operatorname{ext}(\mathcal{X}):= & \{\mathrm{w} \in \mathrm{W}: 10 \mathrm{w} \in \mathcal{X}\} \cup\{\emptyset: 11 \in \mathcal{X}\} \cup \\
& \left\{\operatorname{ext}\left((\mathcal{X})_{\mathrm{w}}\right): \mathrm{w} \in \mathrm{W},(\mathcal{X})_{\mathrm{w}} \neq \emptyset\right\}
\end{aligned}
$$

Further, $\mathcal{X} \simeq \mathcal{W} \mathcal{Y}$ iff $\mathcal{X}, \mathcal{Y} \in \operatorname{Rep}\lceil\mathbb{W} \wedge \operatorname{ext}(\mathcal{X})=\operatorname{ext}(\mathcal{Y})$

Example 5 Subsets of $10 \mathrm{~W} \cup\{11\}$ have depth $\varepsilon$ and code subsets of $\mathrm{W} \cup\{\varnothing\}$. Further, if $x \in 10 \mathrm{~W} \cup\{11\}$, then $w:=\langle\langle\langle x, a\rangle, b\rangle, c\rangle \in$ pair. This word begins with $00^{\prime} 00^{\prime} 00^{\prime} 10 v \ldots$ or $00^{\prime} 00^{\prime} 00^{\prime} 11 \ldots$ and ends with $\ldots 00 c^{*}$. If $w \in \mathcal{X} \in \operatorname{Rep}\lceil\mathbb{W}$, then $\operatorname{ext}\left((\mathcal{X})_{c, b, a}\right) \in \operatorname{ext}\left((\mathcal{X})_{c, b}\right) \in \operatorname{ext}\left((\mathcal{X})_{c}\right) \in \operatorname{ext}(\mathcal{X})$, and $\emptyset \in \operatorname{ext}\left((\mathcal{X})_{c, b, a}\right)$ or $v \in \operatorname{ext}\left((\mathcal{X})_{c, b, a}\right)$, depending on whether $x=11$ or $x=10 v$.

The main step in the construction of a model of $\mathbb{A}(\mathrm{PHCA})$ is to build a suitable model $(\mathcal{W}, \mathcal{S})$ of the arithmetical closure $\mathbb{S}\left(\mathrm{PHCA}^{\natural}\right)$ of $\mathrm{PHCA}^{\natural}$ from a model $\mathcal{W}_{0}$ of $\mathrm{PHCA}^{\natural}$ that preserves the validity of $\mathrm{L}_{p}$ sentences. $(\mathcal{W}, \mathcal{S})$ will be such that for $\mathcal{A}:=\left\{\operatorname{ext}(\mathcal{X}): \mathcal{X} \in \mathcal{S} \cap \operatorname{Rep}^{*}\right\},(\mathcal{W}, \mathcal{A})$ is a model of $\mathbb{A}(\mathrm{PHCA})$. The theory

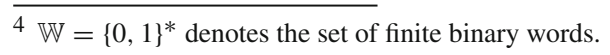


$\mathbb{S}\left(\mathrm{PHCA}^{\natural}\right)$ is formulated in the language $\mathrm{L}_{p}^{2}$ that extends $\mathrm{L}_{p}$ by set terms $S, T, \ldots$ and the elementhood relation $u \in S$. Each set variable $U, V, \ldots$ and $\emptyset$ are set terms, and with $S$, also $(S)_{t}$ and $S^{s, t}$ are set terms. $(S)_{s, t}$ is short for $\left((S)_{s}\right)_{t}$. There will be axioms for set terms stating that the set constant $\emptyset$ has no elements, $s \in(S)_{t}$ iff $\langle s, t\rangle \in S$, and that $r \in S^{s, t}$ iff $r \in S \wedge 00 \times s 0 \nsubseteq r \wedge r \leq t$. Note that for $\vee \in \mathrm{W}$ and $\mathrm{w} \in \mathbb{W}, \mathrm{dth}\left(\mathcal{X}^{\mathrm{w}, \mathrm{v}}\right) \leq \mathrm{w}$ and $\mathrm{wth}\left(\mathcal{X}^{\mathrm{w}, \mathrm{v}}\right) \leq \mathrm{v}$. Also note that wth $\left((S)_{t}\right) \leq \mathrm{wth}(S)$ and wth $\left(S^{s, t}\right) \leq \mathrm{wth}(S)$. The same holds true for the depth.

Subsequently, we often work with the language $L_{p}^{2}(\sim, \mathbb{W})$ whose additional atoms are $S \sim T$ and $\mathbb{W}(s)$ (also written as $s \in \mathbb{W}$ ). The intended interpretation of $\sim$ is that $S$ and $T$ code sets with the same extension, and the relation symbol $\mathbb{W}$ is interpreted by the standard words $\mathbb{W}$. The $\Sigma_{\infty}^{b}$ formulas of $L_{p}$ are lifted canonically to the $\Sigma_{\infty}^{0, b}$ formulas of $\mathrm{L}_{p}^{2}$ and $\mathrm{L}_{p}^{2}(\sim, \mathbb{W})$ : They are generated from the literals of $\mathrm{L}_{p}^{2}$ and $\mathrm{L}_{p}^{2}(\sim, \mathbb{W})$, respectively, as before. Finally, elementary formulas do not contain bound set variables, and $\Sigma$ formulas do not contain universally bound set variables.

To avoid confusion, we stress that the theory $\mathbb{S}\left(\mathrm{PHCA}^{\natural}\right)$ is formulated in the language $L_{p}^{2}$. Yet, we often argue in structures $\left(\mathcal{W}^{\prime}, \mathcal{S}^{\prime}, \simeq^{\prime}, \mathbb{W}\right)$ for a language $L_{p}^{2}(\sim, \mathbb{W})$. Thereby, $\mathcal{W}^{\prime}=\left(\mathrm{W}^{\prime}, \ldots\right)$ is a model of PHCA, and the relation symbol $\mathbb{W}$ is always interpreted by the standard words, and thus henceforth omitted. The second order variables range over $\mathcal{S}^{\prime}$, a collection of subsets of $\mathrm{W}^{\prime}$, and $\simeq^{\prime} \subseteq \mathcal{S}^{\prime} \times \mathcal{S}^{\prime}$ interprets $\sim$. Further, if an $L_{p}^{2}(\sim)$ structure is introduced as $\left(\mathcal{W}_{0}, \mathcal{S}_{0}, \simeq\right)$, then we mean that $\sim$ is interpreted by the restriction of $\simeq \mathcal{W}_{0}$ to $\mathcal{S}_{0}$.

To study some general properties of our coding of sets and to prepare for the subsequent model transformation, we introduce some notations.

(i) $u \in \in_{0} U:=10 u \in U$,

(ii) $V \in \in_{1} U:=(V=\emptyset \wedge 11 \in U) \vee \exists x\left((U)_{x} \neq \emptyset \wedge V \sim(U)_{x}\right)$,

(iii) $U={ }_{0} V:=\forall x\left[x \in_{0} U \leftrightarrow x \in_{0} V\right]$,

(iv) $\left(\forall X \in \in_{1} U\right) A(X):=(11 \in U \rightarrow A(\emptyset)) \wedge \forall x\left[(U)_{x} \neq \emptyset \rightarrow A\left((U)_{x}\right)\right]$,

(v) $\left(\exists X \in_{1} U\right) A(X):=(11 \in U \wedge A(\emptyset)) \vee \exists x\left[(U)_{x} \neq \emptyset \wedge A\left((U)_{x}\right)\right]$,

(vi) $U={ }_{1} V:=\left(\forall X \in_{1} U\right)\left(X \in_{1} V\right) \wedge\left(\forall X \in_{1} V\right)\left(X \in_{1} U\right)$.

With the aim to turn elementary $\mathrm{L}_{p}^{2}(\sim, \mathbb{W})$ formulas into $\Sigma_{\infty}^{0, b}$ formulas of $\mathrm{L}_{p}^{2}(\sim, \mathbb{W})$, we denote by $A^{v}$ the formula obtained from $A$ by replacing each unbounded word quantifier $\mathcal{Q} x B$ by $(\mathcal{Q} x \leq v) B$. And to get rid of the relation symbol $\sim$, we say that for $\mathrm{w} \in \mathbb{W}, A_{\mathrm{W}}$ is obtained from $A$ by replacing each expression $S \sim T$ in $A$ by $E_{\mathrm{W}}(S, T)$, where $E_{\varepsilon}(U, V):=U=_{0} V$ and $E_{\mathrm{W} i}(U, V):=\left(U==_{0} V\right) \wedge\left(U={ }_{1} V\right)_{\mathrm{W}}(i \in\{0,1\})$. $A_{\mathrm{W}, v}$ is obtained from $A$ by replacing $S \sim T$ by $E_{\mathrm{w}}^{v}(S, T)$ ) (i.e. $\left.\left(E_{\mathrm{W}}(S, T)\right)^{v}\right)$. Also the following abbreviations prove convenient:

$$
\begin{aligned}
\left(\forall x \in_{0} U\right)^{v} A(x) & :=(\forall x \leq v)[10 x \in U \rightarrow A(x)] \\
\left(\forall X \in_{1} U\right)^{v} A(X) & :=(11 \in U \rightarrow A(\emptyset)) \wedge(\forall x \leq v)\left[\left((U)_{x} \neq \emptyset\right)^{v} \rightarrow A\left((U)_{x}\right)\right] ;
\end{aligned}
$$

( $\left.\exists x \in_{0} U\right)^{v} A(x)$ and $\left(\exists X \in_{1} U\right)^{v} A(X)$ are defined analogously. The following is now readily checked by induction on $\mathrm{W} \in \mathbb{W}$ : 
Lemma 6 Let $\mathrm{w} \in \mathbb{W}$. For all $\mathcal{X}, \mathcal{Y} \in \operatorname{Rep}\left\lceil\mathrm{w}, \mathcal{X} \simeq \mathcal{Y}\right.$ iff $E_{\mathrm{w}}(\mathcal{X}, \mathcal{Y})$. If in addition wth $(\mathcal{X}, \mathcal{Y}) \leq \mathrm{v}$, then $\mathcal{X} \simeq \mathcal{Y}$ iff $E_{\mathrm{w}}^{\mathrm{v}}(\mathcal{X}, \mathcal{Y})$. Further, for each elementary $\mathrm{L}_{p}^{2}(\sim)$ formula $A(\vec{U})$, and all $\overrightarrow{\mathcal{X}} \in \operatorname{Rep} \mid \mathrm{w}$ with $\mathrm{wth}(\overrightarrow{\mathcal{X}}) \leq \mathrm{v}$, we have $A(\overrightarrow{\mathcal{X}}) \Leftrightarrow A_{\mathrm{w}, \mathrm{v}}(\overrightarrow{\mathcal{X}})$.

With regard to the definition of $\mathbb{S}\left(\mathrm{PHCA}^{\natural}\right)$ we state the following observation:

Lemma 7 If $\left(\mathcal{W}, \operatorname{Rep} \mid \mathbb{W}, \simeq^{\prime}\right) \models \forall X, Y\left(X \sim Y \leftrightarrow X={ }_{0} Y \wedge X={ }_{1} Y\right)$, then $\simeq^{\prime}$ and $\simeq$ coincide on $\operatorname{Rep}\lceil\mathbb{W}$.

As a next step, we consider the $\mathrm{L}_{p}^{2}$ theory $\mathbb{S}\left(\mathrm{PHCA}^{\natural}\right)$ that comprises the aforementioned axioms for set terms. Further, $\mathbb{S}\left(\mathrm{PHCA}^{\natural}\right)$ inherits all axioms of $\mathrm{PHCA}^{\natural}$ with the exception of induction, features $\Sigma_{\infty}^{0, b}$-comprehension, set induction and bounded collection lifted to $\Sigma_{\infty}^{0, b}$ formulas, and comprises the schema of finite $\Sigma_{\infty}^{0, b}$ choice, in symbols $\left(\Sigma_{\infty}^{0, b}-\mathrm{AC}^{b}\right)$ : For each $\Sigma_{\infty}^{0, b}$ formula $A(U, u, v)$ of $\mathrm{L}_{p}^{2}$,

$$
(\forall x \leq t) \exists X \exists y A(X, x, y) \rightarrow \exists X \exists y(\forall x \leq t)(\exists z \leq y) A\left((X)_{x}, x, z\right) .
$$

That $\mathrm{PHCA}^{\natural}$ and $\mathbb{S}\left(\mathrm{PHCA}^{\natural}\right)$ prove the same $\mathrm{L}_{p}$ sentences follows by the next lemma.

Lemma 8 Let $\mathcal{W}_{0}$ be a model of $\mathrm{PHCA}{ }^{\natural}$. Then there is a model $(\mathcal{W}, \mathcal{S})$ of $\mathbb{S}\left(\mathrm{PHCA}^{\natural}\right)$ so that $\mathcal{W} \succ \mathcal{W}_{0}$ is an elementary extension of $\mathcal{W}_{0}$ and further, if $B$ is a $\Sigma$ formula of $\mathrm{L}_{p}^{2}(\sim, \mathbb{W})$ that contains $\mathbb{W}$ only positively and has the property that for each $\mathcal{Z} \subseteq \mathbb{W},(\mathcal{W}, \mathcal{S}, \simeq) \models B[\mathcal{Z} / \mathbb{W}]$ iff $\left(\mathcal{W}, \mathcal{S}, \simeq^{\prime}\right) \models B[\mathcal{Z} / \mathbb{W}]$ whenever $\simeq^{\prime}$ and $\simeq$ agree on $\mathcal{S} \cap \operatorname{Rep}^{\mathcal{W}} \uparrow \mathbb{W}$, then

$$
(*) \quad(\mathcal{W}, \mathcal{S}, \simeq)=B \rightarrow(\exists b \in \mathbb{W}) B[\{\mathrm{w}: \mathrm{w} \leq b\} / \mathbb{W}] .
$$

Proof Assume that $\mathcal{W}_{0}$ is a model of $\mathrm{PHCA}^{\natural}$. To obtain a suitable expansion $\left(\mathcal{W}, \mathcal{S}^{\prime}, \simeq^{\prime}\right)$ of $\mathcal{W}_{0}$ that meets $(*)$, we let $T$ be the union of the six sets of formulas listed below. $T$ is finitely realizable, i.e. for each finite subset $G \subseteq T$, there is a structure $\left(\mathcal{W}^{\prime}, \mathcal{S}^{\prime}, \simeq^{\prime}\right)$ and $\mathrm{c} \in \mathrm{W}^{\prime}, \mathcal{F} \in \mathcal{S}^{\prime}$, so that for each formula $C(\mathrm{P}, \mathrm{p}) \in G,\left(\mathcal{W}^{\prime}, \mathcal{S}^{\prime}, \simeq^{\prime}\right) \models C(\mathcal{F}, \mathrm{c})$. Below, $\left(A_{i}(u, v): i \in \mathbb{N}\right)$ is an enumeration of the formulas of $\mathrm{L}_{p}(\sim, \mathrm{P}, \mathrm{p})$ with free variables $u$, $v$, and for each $j \in \mathbb{N},\left(B_{i, j}\left(U, u_{1}, \ldots, u_{j}, v\right): i \in \mathbb{N}\right)$ is an enumeration of the $\Sigma_{\infty}^{0, b}$ formulas of $\mathrm{L}_{p}^{2}$ with free variables $U, u_{1}, \ldots, u_{j}, v$. Further, we let $s \in(\mathrm{P})_{<t}$ be a shorthand for the formula $(\exists x, y \leq s)\left(s=\langle x, y\rangle \wedge y<t \wedge x \in(\mathrm{P})_{y}\right)$.

(i) $\{\bar{w} \leq \mathrm{p}: w \in \mathbb{W}\}^{5}$ and $\left\{A: \mathcal{W}_{0} \models A, A\right.$ an $\mathrm{L}_{p}\left(c_{w}: w \in \mathbb{W}_{0}\right)$ sentence $\}$,

(ii) $\left\{\forall x\left[(\exists z \leq \mathrm{p}) A_{i}(x, z) \rightarrow \exists z\left(A_{i}(x, z) \wedge(\forall y<z) \neg A_{i}(x, y)\right)\right]: i \in \mathbb{N}\right\}$,

(iii) $\left\{(\forall z \leq \mathrm{p}) \forall x \exists y\left[\left\{w: B_{i, 2}\left((\mathrm{P})_{<0 \times z}, x, w\right)\right\}=(\mathrm{P})_{0 \times z, y}\right]: i \in \mathbb{N}\right\}$,

(iv) $\left\{(\forall z \leq \mathrm{p}) \forall b, c\left[(\forall x \leq b) \exists y B_{i, 3}\left((\mathrm{P})_{<0 \times z}, x, y, c\right) \rightarrow\right.\right.$

$$
\left.\left.\exists a(\forall x \leq b)(\exists y \leq a) B_{i, 3}\left((\mathrm{P})_{<0 \times z}, x, y, c\right)\right]: i \in \mathbb{N}\right\},
$$

(v) $\forall X, Y\left(\operatorname{dth}(X, Y) \leq \mathrm{p} \rightarrow\left(X \sim Y \leftrightarrow X==_{0} Y \wedge X={ }_{1} Y\right)\right)$,

(vi) $\forall X[\varepsilon \in X \wedge \forall x(x \in X \rightarrow x 0 \in X \wedge x 1 \in X) \rightarrow \forall x(x \in X)]$.

\footnotetext{
${ }^{5}$ For each $w \in \mathbb{W}$, we have that $\bar{w}$ is the canonical closed $L$ term designating $w$.
} 
Since the theory $T$ is finitely realizable, compactness provides a structure $\left(\mathcal{W}, \mathcal{S}^{\prime}, \simeq^{\prime}\right)$ and $\mathrm{c} \in \mathrm{W}, \mathcal{F} \in \mathcal{S}^{\prime}$ so that $\left(\mathcal{W}, \mathcal{S}^{\prime}, \simeq^{\prime}\right) \models C(\mathcal{F}, \mathrm{c})$ for each $C(\mathrm{P}, \mathrm{p}) \in T$. By (i) we have that $\mathrm{w}$ is non-standard and that $\mathcal{W}_{0} \prec \mathcal{W}$, (ii) tells us that each non-empty subclass of $\{w: w \leq c\}$ which is $L_{p}$-definable with parameters from $W \cup\{\mathcal{F}\}$ has a <-minimal element, (iii) states that $(\mathcal{F})_{0 \times z}$ contains in particular all the sets that are definable by a $\Sigma_{\infty}^{0, b}$ formula of $\mathrm{L}_{p}^{2}$ with word parameters and set parameters from $(\mathcal{F})_{<0 \times z}$, (iv) guarantees bounded collection, (v) inductively defines the relation for sets whose depth is bounded by $\mathrm{C}$ and (vi) asserts set induction. We claim that for

$$
\mathcal{S}:=\left\{\mathcal{Z}: \mathcal{Z}=(\mathcal{F})_{0 \times b, e}, b \in \mathbb{W}, e \in \mathbb{W}\right\}
$$

$(\mathcal{W}, \mathcal{S})$ is a model of $\mathbb{S}\left(\mathrm{PHCA}^{\natural}\right)$. Due to the definition of $\mathcal{S},(\mathcal{W}, \mathcal{S})$ satisfies $\Sigma_{\infty}^{0, b}$ comprehension. It remains to show that $\left(\Sigma_{\infty}^{0, b}-\mathrm{AC}^{b}\right)$ is satisfied. Let $B(U, u, v)$ be $\Sigma_{\infty}^{0, b}, \mathrm{t} \in \mathrm{W}$ and assume that for each word $\mathrm{w} \leq \mathrm{t}$, there are $\mathcal{Y} \in \mathcal{S}, \mathrm{y} \in \mathrm{W}$ so that $(\mathcal{W}, \mathcal{S}) \models B(\mathcal{Y}, \mathrm{w}, \mathrm{y})$. Thus,

$$
\left(\mathcal{W}, \mathcal{S}^{\prime}\right) \models(\forall w \leq \mathrm{t})(\exists b \in \mathbb{W}) \exists e, y B\left((\mathcal{F})_{0 \times b, e}, w, y\right) .
$$

By choice of $\mathcal{F}$ and $\mathrm{c}$,

$$
\emptyset \neq \mathcal{X}:=\left\{b \leq^{\mathcal{W}} \mathrm{c}:\left(\mathcal{W}, \mathcal{S}^{\prime}\right) \models(\forall w \leq \mathrm{t}) \exists e, y B\left((\mathcal{F})_{0 \times b, e}, w, y\right)\right\}
$$

has a $\leq$-minimal element of the form $0 \times b_{0}$. Because $\{w \leq \mathcal{W} \mathrm{c}: w \notin \mathbb{W}\} \subseteq \mathcal{X}$ has no $\leq$-minimal element, $b_{0} \in \mathbb{W}$. Bounded collection provides a word $\mathrm{S}$ so that $(\forall w \leq \mathrm{t})(\exists e, y \leq \mathrm{s}) B\left((\mathcal{F})_{0 \times b_{0}, e}, w, y\right)$. Then,

$$
\begin{array}{r}
\mathcal{Z}:=\left\{\langle z, w\rangle:(\exists e \leq \mathrm{s})\left[(\exists y \leq \mathrm{s}) B\left((\mathcal{F})_{0 \times b_{0}, e}, w, y\right) \wedge\right.\right. \\
\left.\left.\left(\forall e^{\prime} \leq \mathrm{s}\right)\left(e^{\prime}<_{\operatorname{lex}} e \rightarrow(\forall y \leq \mathrm{s}) \neg B\left((\mathcal{F})_{0 \times b_{0}, e^{\prime}}, w, y\right)\right) \wedge z \in(\mathcal{F})_{0 \times b_{0}, e}\right]\right\}
\end{array}
$$

is in $\mathcal{S}$ and $(\mathcal{W}, \mathcal{S}) \models(\forall w \leq \mathrm{t})(\exists y \leq \mathrm{s}) B\left((\mathcal{Z})_{w}, w, y\right)$.

To show $(*)$, assume that $(\mathcal{W}, \mathcal{S}, \simeq) \models B$ and that for each $\mathcal{Z} \subseteq \mathbb{W}$, the truth of $B[\mathcal{Z} / \mathbb{W}]$ only depends on the interpretation of $\sim$ on $\mathcal{S} \cap \operatorname{Rep}^{\mathcal{W}} \uparrow \mathbb{W}$. By (v) and Lemma 7 it follows that $\simeq^{\prime}$ and $\simeq \mathcal{W}$ agree on $\mathcal{S}^{\prime} \cap \operatorname{Rep}^{\mathcal{W}} \uparrow \mathbb{W}$. Hence, $\left(\mathcal{W}, \mathcal{S}, \simeq^{\prime}\right) \models B$. Let $B^{\prime}$ be the formula obtained form $B$ by replacing each expression $\exists Y A(Y)$ by $\left.(\exists b \in \mathbb{W}) \exists e A\left((\mathcal{F})_{0 \times b, e}\right)\right)$. Then we have that $\left(\mathcal{W}, \mathcal{S}^{\prime}, \simeq^{\prime}\right) \models B^{\prime}$. With $B$, also $B^{\prime}$ contains $\mathbb{W}$ only positively, since $B$ is $\Sigma$. Arguing as before in $\left(\mathcal{W}, \mathcal{S}^{\prime}, \simeq^{\prime}\right)$ yields that

$$
\mathrm{b}_{0}:=\min _{\sqsubseteq}\left\{0 \times \mathrm{b}: \mathrm{b} \leq^{\mathcal{W}} \mathrm{c} \wedge B^{\prime}[\{\mathrm{w}: \mathrm{w} \leq \mathrm{b}\} / \mathbb{W}]\right\}
$$

exists and is in $\mathbb{W}$. By persistence, also $\left(\mathcal{W}, \mathcal{S}, \simeq^{\prime}\right) \models B\left[\left\{\mathrm{w}: \mathrm{w} \leq \mathrm{b}_{0}\right\} / \mathbb{W}\right]$. By assumption, $(\mathcal{W}, \mathcal{S}, \simeq) \models B\left[\left\{\mathrm{w}: \mathrm{w} \leq \mathrm{b}_{0}\right\} / \mathbb{W}\right]$.

The structure $(\mathcal{W}, \mathcal{S})$ constructed in the previous proof gives rise to an $\mathcal{L}^{*}$ structure $\mathcal{M}=(\mathcal{W}, \mathcal{A})$. We set $\mathcal{A}:=\left\{\operatorname{ext}(\mathcal{X}): \mathcal{X} \in \mathcal{S} \cap \mathrm{Rep}^{*}\right\}$ and extend the interpretation of the function and relation symbols of $\mathrm{L}_{p}$ to the new domain $\mathrm{W} \cup \mathcal{A}$ as follows: If $\overrightarrow{\mathrm{y}} \in \mathrm{W} \cup \mathcal{A}$ is not a sequence of words, then $f^{\mathcal{M}}(\overrightarrow{\mathrm{y}}):=\varepsilon$ and $R^{\mathcal{M}}(\overrightarrow{\mathrm{y}}):=\perp$. 
Theorem $9 \mathcal{M}:=(\mathcal{W}, \mathcal{A})$ is a model of $\mathbb{A}\left(\mathrm{PHCA}^{\natural}\right)$. Further, $\mathrm{PHCA}^{\natural}$ and $\mathbb{A}\left(\mathrm{PHCA}^{\natural}\right)$ prove the same $\mathrm{L}_{p}$ formulas.

The second claim is immediate from the fact that $\mathcal{W} \models \mathrm{PHCA}^{\natural}$. To verify that $\mathcal{M}$ satisfies all axioms of $\mathbb{A}\left(\mathrm{PHCA}^{\natural}\right)$ we have to resort to the underlying structure $\mathcal{S}:=(\mathcal{W}, \mathcal{S}, \simeq)$ constructed in the previous proof. Depending on a function $\sigma$ that maps a variable $u_{i}$ of $\mathcal{L}^{*}$ either to the word variable $u_{i}$ or the set variable $U_{i}$ of $\mathrm{L}_{p}^{2}$, we assign to each formula $A$ of $\mathcal{L}^{*}$ a formula $A^{\sigma}$ of the language $\mathrm{L}_{p}^{2}(\sim, \mathbb{W})$. If $\mathrm{FV}(A)=\left\{u_{1}, \ldots, u_{n}\right\}$, then $\mathrm{FV}\left(A^{\sigma}\right) \subseteq\left\{\sigma\left(u_{1}\right), \ldots, \sigma\left(u_{n}\right), v_{1}, \ldots, v_{n}\right\}$. The idea is that $v_{i}$ is a bound for the width of $\sigma\left(u_{i}\right)=U_{i}$. If $\xi \in\{u, U\}$, then $\sigma[\xi](u):=\xi$ and $\sigma[\xi](v):=\sigma(v)$.

(i) $u^{\sigma}:=\sigma(u)$, and if $t$ is an $\mathcal{L}^{*}$ term other than a variable, then $t^{\sigma}:=\varepsilon$ if $\sigma(u)=U$ for some $u \in \mathrm{FV}(t)$, and $t^{\sigma}:=t$ otherwise.

(ii) If $R$ is a relation symbol of $\mathrm{L}_{p}$, then $\left(R\left(t_{1}, \ldots, t_{m}\right)\right)^{\sigma}:=\perp$ if $t_{1}^{\sigma}, \ldots, t_{m}^{\sigma}$ contains a set variable, and $R\left(t_{1}^{\sigma}, \ldots, t_{m}^{\sigma}\right)$ otherwise. $(\mathrm{W}(t))^{\sigma}:=\top$ if $t^{\sigma}$ is not a set variable, and $\perp$ otherwise. $(\mathrm{S}(t))^{\sigma}:=\neg(\mathrm{W}(t))^{\sigma}$.

(iii) If $t^{\sigma}$ is not a set variable, then $(s \in t)^{\sigma}:=\perp$. Otherwise, assume $t^{\sigma}=U_{i}$. If $s^{\sigma}$ is not a set variable, then $\left(s \in U_{i}\right)^{\sigma}:=s^{\sigma} \in_{0} U_{i}$, and if $s^{\sigma}=U_{j}$, then $(s \in t)^{\sigma}:=\left(U_{j} \in U_{1} U_{i}\right)^{v_{i}}$.

(iv) $(\neg A)^{\sigma}:=\neg A^{\sigma}$ and $(A \& B)^{\sigma}:=A^{\sigma} \& B^{\sigma}$, where $\& \in\{\wedge, \vee\}$.

(v) If $t^{\sigma}$ is not a set variable, then $((\exists x \in t) A[x / u])^{\sigma}:=\perp$. And if $t^{\sigma}=U_{i}$, then

$$
((\exists x \in t) A[x / u])^{\sigma}:=\left(\exists x \in_{0} U_{i}\right)^{v_{i}} A^{\sigma[u]}[x / u] \vee\left(\exists X \in_{1} U_{i}\right)^{v_{i}} A^{\sigma[U]}[X / U] .
$$

(vi) If $t^{\sigma}$ is not a set variable, then $((\forall x \in t) A[x / u])^{\sigma}:=\top$. And if $t^{\sigma}=U_{i}$, then

$$
((\forall x \in t) A[x / u])^{\sigma}:=\left(\forall x \in \in_{0} U_{i}\right)^{v_{i}} A^{\sigma[u]}[x / u] \wedge\left(\forall X \in_{1} U_{i}\right)^{v_{i}} A^{\sigma[U]}[X / U] .
$$

(vii) Finally, $(\exists x A[x / u])^{\sigma}$ and $(\forall x A[x / u])^{\sigma}$ are defined as follows:

$$
\begin{aligned}
& \left.\exists x A^{\sigma[u]}[x / u] \vee \exists X \exists y(\exists b \in \mathbb{W}) A^{\sigma[U]}\left[X^{b, y} / U, y / v\right]\right), \\
& \left.\forall x A^{\sigma[u]}[x / u] \wedge \forall X \forall y(\forall b \in \mathbb{W}) A^{\sigma[U]}\left[X^{b, y} / U, y / v\right]\right) .
\end{aligned}
$$

It is readily observed that this translation has the following properties.

Lemma 10 Suppose that $\sigma(\vec{u})=\vec{U}, \sigma(\vec{w})=\vec{w}$ and that $A(\vec{u}, \vec{w})$ is an $\mathcal{L}^{*}$ formula with the displayed variables free. Then we have for all $\overrightarrow{\mathcal{X}} \in \operatorname{Rep}^{*} \cap \mathcal{S}, \vec{a}:=$ $\operatorname{ext}(\overrightarrow{\mathcal{X}}), \overrightarrow{\mathrm{v}}, \overrightarrow{\mathrm{w}} \in \mathrm{W}$ with $\mathrm{wth}(\overrightarrow{\mathcal{X}}) \leq \overrightarrow{\mathrm{v}}$,

$$
\mathcal{M} \models A(\overrightarrow{\mathrm{a}}, \overrightarrow{\mathrm{w}}) \quad \Longleftrightarrow \quad(\mathcal{W}, \mathcal{S}, \simeq) \models A^{\sigma}(\overrightarrow{\mathcal{X}}, \overrightarrow{\mathrm{w}}, \overrightarrow{\mathrm{v}}) .
$$

If $A$ is $\Delta_{0}$, then $A^{\sigma}$ is $\Sigma_{\infty}^{0, b}$ and $\mathbb{W}$-free, and if $A$ is $\Sigma$, then $A^{\sigma}$ contains $\mathbb{W}$ only positively.

Proof [Theorem] For notational convenience, it is henceforth assumed that the displayed formulas do not contain additional set and number parameters. The handling 
of parameters does not cause any additional problems. Only when turning elementary formula $A$ of $\mathrm{L}_{p}^{2}(\sim)$ into $\Sigma_{\infty}^{0, b}$ formulas $A_{\mathrm{b}, \mathrm{v}}$ of $\mathrm{L}_{p}^{2}$, we have to take care that $\mathrm{b}$ and $\mathrm{v}$ are also bounds for the depths and widths of the set parameters. Below, we reason in the structure $(\mathcal{W}, \mathcal{S}, \simeq)$.

That $\mathcal{M} \models \vec{x} \in \mathrm{W} \rightarrow A^{\mathrm{W}}(\vec{x})$ for each axiom $A(\vec{u})$ of $\mathrm{PHCA}^{\natural}$ is immediate by the construction of $\mathcal{M}$. The same holds for the regularity axiom. If $\mathrm{w}$ is a word, then $\{10 \mathrm{v}: \mathrm{v} \leq \mathrm{W}\}$ represents the set claimed by (W.1). And if $A(u, w)$ is a $\Delta_{0}$ formula of $\mathcal{L}^{*}$ (here and only once we exemplarily deal with a set parameter $u$ ), $a=$ $\operatorname{ext}(\mathcal{X}), \operatorname{dth}(\mathcal{X}) \leq \mathrm{b}$ and $\operatorname{wth}(\mathcal{X}) \leq \mathrm{v}$, then, for $B(U, w, v):=A^{\sigma[U, w]}$, Lemma 10 and Lemma 6 yield that

$$
\{\mathrm{w} \in \mathrm{W}: A(\mathrm{a}, \mathrm{w})\}=\{\mathrm{w}: B(\mathcal{X}, \mathrm{w}, \mathrm{v})\}=\left\{\mathrm{w}: B_{\mathrm{b}, \mathrm{v}}(\mathcal{X}, \mathrm{w}, \mathrm{v})\right\}
$$

As $\mathbb{S}\left(\mathrm{PHCA}^{\natural}\right)$ is equipped with $\Sigma_{\infty}^{0, b}$-comprehension, $\Delta_{0}$-induction in $\mathcal{M}$ holds since set induction holds in $(\mathcal{W}, \mathcal{S})$.

The Kripke-Platek axioms are easily checked, too: If $\mathrm{a}=\operatorname{ext}(\mathcal{X})$ and $\mathrm{b}=\operatorname{ext}(\mathcal{Y})$, then $\operatorname{ext}(\mathcal{Z})=\{\mathrm{a}, \mathrm{b}\}$ for $\mathcal{Z}:=\{\langle x, 0\rangle,\langle y, 1\rangle: x \in \mathcal{X}, y \in \mathcal{Y}\} \cup \mathcal{Z}^{\prime}$, where $\mathcal{Z}^{\prime}=\{11\}$ if $\mathcal{X}$ or $\mathcal{Y}$ are empty and $\emptyset$ otherwise. And if e.g. $\mathrm{w} \in \mathrm{W}$ and $\mathrm{b}:=\operatorname{ext}(\mathcal{Y}) \neq \emptyset$, then $\operatorname{ext}(\mathcal{Z})=\{\mathrm{w}, \mathrm{b}\}$ for $\mathcal{Z}:=\{10 \mathrm{w},\langle y, 1\rangle: y \in \mathcal{Y}\}$. Further, if a $=\operatorname{ext}(\mathcal{X})$ with wth $(\mathcal{X}) \leq \mathrm{v}$, then $\operatorname{ext}(\mathcal{Z})=\bigcup$ a for $\left.\mathcal{Z}:=\left\{h(z, y): y \leq \mathrm{v} \wedge z \in(\mathcal{X})_{y}\right)\right\}$, where $h(z, y)=z$ if $z \notin$ pair, and $h(z, y)=\left\langle z_{0}, z_{1}^{*} 00 y^{*}\right\rangle$ if $z=\left\langle z_{0}, z_{1}\right\rangle .{ }^{6}$ If $A(u)$ is $\Delta_{0}, \mathrm{a}=\operatorname{ext}(\mathcal{X}), \operatorname{dth}(\mathcal{X}) \leq \mathrm{b}$ and $\operatorname{wth}(\mathcal{X}) \leq \mathrm{v}$, then let $B_{0}(u):=A^{\sigma[u]}$ and $B_{1}(U, v):=A_{\mathrm{b}, \mathrm{v}}^{\sigma[U]}$. Then the set $\{\mathrm{z} \in \mathrm{a}: A(\mathrm{z})\}$ is now represented by the set $\mathcal{Z}$ given as

$$
\left\{\mathrm{w}: \mathrm{w} \in_{0} \mathcal{X} \wedge B_{0}(\mathrm{w})\right\} \cup\left\{\langle\mathrm{w}, \mathrm{x}\rangle \in \mathcal{X}: B_{1}\left((\mathcal{X})_{\mathrm{x}}, \mathrm{v}\right)\right\} \cup\left\{11: B_{1}(\emptyset, \mathrm{v})\right\}
$$

Finally, towards the verification of $\Delta_{0}$ collection, suppose that $A\left(u_{0}, u_{1}\right)$ is a $\Delta_{0}$ formula of $\mathcal{L}^{*}$ and that $\mathrm{a}=\operatorname{ext}(\mathcal{X}), \operatorname{dth}(\mathcal{X}) \leq \mathrm{b}$, and wth $(\mathcal{X}) \leq \mathrm{v}$. By Lemma 10 , $\mathcal{M} \models(\forall x \in \mathrm{a}) \exists b A(x, b)$ iff

$$
\begin{aligned}
& \left(\forall x \in_{0} \mathcal{X}\right)^{\mathrm{v}}\left(\exists y A^{\sigma\left[u_{0}, u_{1}\right]}\left[x / u_{0}, y / u_{1}\right] \vee\right. \\
& \left.\quad \exists Y \exists z(\exists \mathrm{b} \in \mathbb{W}) A^{\sigma\left[u_{0}, U_{1}\right]}\left[x / u_{0}, Y^{\mathrm{b}, z} / U_{1}, z / v_{1}\right]\right) \quad \text { and } \\
& \left(\forall X \in_{1} \mathcal{X}\right)^{\mathrm{v}}\left(\exists y A^{\sigma\left[U_{0}, u_{1}\right]}\left[X / U_{0}, y / u_{1}, \mathrm{v} / v_{0}\right] \vee\right. \\
& \left.\quad \exists Y \exists z(\exists \mathrm{b} \in \mathbb{W}) A^{\sigma\left[U_{0}, U_{1}\right]}\left[X / U_{0}, Y^{\mathrm{b}, z} / U_{1}, \mathrm{v} / v_{0}, z / v_{1}\right]\right),
\end{aligned}
$$

which is easily seen to be logically equivalent to

$$
(\forall x \leq \mathrm{v}) \exists Y \exists z(\exists \mathrm{b} \in \mathbb{W}) \exists y C\left(\mathcal{X}, x, Y^{\mathrm{b}, z}, y\right),
$$

for some $\Sigma_{\infty}^{0, b}$ formula $C(U, u, V, v)$ of $\mathrm{L}_{p}^{2}(\sim)$. Since $(*)$ is $\Sigma$, contains $\mathbb{W}$ only positively and depends only on the interpretation of $\sim$ on $\mathcal{S} \cap$ Rep* , Lemma 8 provides

\footnotetext{
${ }^{6}$ Concerning the role of the function $h$, observe that $\operatorname{ext}(\{\langle\langle 100,0\rangle, 0\rangle,\langle\langle 101,0\rangle, 1\rangle\})=\{\{\{0\}\},\{\{1\}\}\}$, but $\operatorname{ext}(\{\langle 100,0\rangle,\langle 101,0\rangle\})=\{\{0,1\}\}$.
} 
$\mathrm{a} \mathrm{b}_{0} \in \mathbb{W}$ with $\mathrm{dth}(\mathcal{X}) \leq \mathrm{b}_{0}$ so that, using Lemma 6 ,

$$
(\forall x \leq \mathrm{v}) \exists Y(\exists z \geq \mathrm{v}) \exists y C_{\mathrm{b}_{0}, z}\left(\mathcal{X}, x, Y^{\mathrm{b}_{0}, z}, y\right) .
$$

As $C_{\mathrm{b}_{0}, z}$ is a $\Sigma_{\infty}^{0, b}$ formula of $\mathrm{L}_{p}^{2}$, the finite choice axiom $\left(\Sigma_{\infty}^{0, b}-\mathrm{AC}^{b}\right)$ provides $\mathcal{Z} \in \mathcal{S}$ and $z \in \mathrm{W}$ so that

$$
(\forall x \leq \mathrm{v})(\exists y \leq \mathrm{z})\left[\mathrm{wth}\left((\mathcal{Z})_{x}\right) \leq \mathrm{z} \wedge \mathrm{dth}\left((\mathcal{Z})_{x}\right) \leq \mathrm{b}_{0} \wedge C_{\mathrm{b}_{0}, \mathrm{z}}\left(\mathcal{X}, x,(\mathcal{Z})_{x}, y\right)\right]
$$

For each $x \leq \mathrm{v}$, the width and depth of $(\mathcal{Z})_{x}$ are bounded by $\mathrm{z}$ and $\mathrm{b}_{0}$, respectively. Let $\mathbf{z}^{\prime}:=\langle\mathbf{z}, \mathbf{v}\rangle$ and $\mathcal{Z}^{\prime}:=\mathcal{Z}^{\mathrm{b}_{0} 0, \mathbf{z}^{\prime}}$. Then $(\forall x \leq \mathrm{v})\left[(\mathcal{Z})_{x}=\left(\mathcal{Z}^{\prime}\right)_{x}\right]$ and $\mathcal{Z}^{\prime} \in \mathcal{S} \cap \operatorname{Rep}^{*}$. Hence

$$
(\forall x \leq \mathrm{v})\left(\exists Y \in \in_{1} \mathcal{Z}^{\prime} \cup\{11\}\right)(\exists y \leq \mathrm{z}) C_{\mathrm{b}_{0}, \mathrm{z}}(\mathcal{X}, x, Y, y)
$$

Using Lemma 10, it follows that for the set c := ext $\left(\mathcal{Z}^{\prime} \cup\{11\} \cup\{10 x: x \leq z\}\right), \mathcal{M} \models$ $(\forall x \in \mathrm{a})(\exists b \in \mathrm{c}) A(x, b)$. This concludes our proof.

The previous theorem together with Lemma 1 readily entails the main theorem of this section.

Theorem $11 \mathrm{PHCA}$ and $\mathbb{A}(\mathrm{PHCA})$ prove the same $\forall \exists \Sigma_{\infty}^{b}$ sentences.

\section{Concluding remarks}

We have studied two natural weak admissible set theories over the two base theories PTCA and PHCA, featuring that the collection of all subwords of a given word forms a set, (W.0), and the collection of all words whose length is less than or equal to the length of a given word forms a set, (W.1), respectively. We have proved that the admissible closures $\mathbb{A}(\mathrm{PTCA})$ and $\mathbb{A}(\mathrm{PHCA})$ are conservative over PTCA and PHCA for $\forall \exists \Sigma_{1}^{b}$ and $\forall \exists \Sigma_{\infty}^{b}$ formulas, respectively. Thus, the $\Sigma_{1}^{b}$ definable functions of $\mathbb{A}(\mathrm{PTCA})$ are the polytime functions and the $\Sigma_{\infty}^{b}$ definable functions of $\mathbb{A}$ (PHCA) are the functions in the polynomial time hierarchy.

A set existence axiom similar in spirit to the axiom (W.1) has recently been proposed and studied in the context of Feferman's explicit mathematics [6,7], see Spescha [18], Spescha and Strahm [19,20], and Probst [15]. The systems of explicit mathematics based on (W.1) are based on purely positive comprehension principles. This is in contrast to the set-theoretic framework considered in this article, where our theories feature full $\Delta_{0}$ separation and $\Delta_{0}$ collection.

Let us conclude this article by mentioning two quite different approaches to weak set theories due to Sazonov [17] and Sato [16].

In his program of Bounded Set Theory (BST), Sazonov [17] considers set theories formulated on the basis of a so-called $\Delta$ language, which extends the pure language of set theory by further constructs such as, for example, least fixed points and collapsing. Inspired by results from finite model theory, specific $\Delta$ languages correspond to various complexity classes defined over the hereditarily finite sets. 
In his very recent and extensive work on the role of extensionality in various set theories, Sato [16] studies a rich family of finite set theories and their relationship to classes of computational complexity. The characterization of the latter is inspired by the Cook and Nguyen approach via a two-sorted version of bounded arithmetic [5]. Sato's set theories are urelement-free and based on a core system including, for example, fibers, collapsing and a form of $\Delta_{1}$ separation.

In contrast to these two settings, our approach starts off from well-known systems of first-order bounded arithmetic considered as axioms about urelements, and extends them by admissible closures in the usual language of set theory with urelements, where various set forming principles for collections of urelements are taken into account. Thus, our set up is more similar to the one considered in Jäger [13].

Acknowledgments The research of the first author is supported by the Alexander von Humboldt Foundation. The second author is grateful to the Department of Computer Science of the University of Swansea for its hospitality while preparing the final version of this paper.

\section{References}

1. Buss, S.R.: Bounded Arithmetic. Bibliopolis, Napoli (1986)

2. Buss, S.R.: A conservation result concerning bounded theories and the collection axiom. Proc. Am. Math. Soc. 100(4), 709-715 (1987)

3. Cantini, A.: Asymmetric interpretation for bounded theories. Math. Logic Quart. 42, 270-288 (1996)

4. Cobham, A.: The intrinsic computational difficulty of functions. In: Logic, Methodology and Philosophy of Science II. pp. 24-30. North Holland, Amsterdam (1965)

5. Cook, S.A., Nguyen, P.: Logical Foundations of Proof Complexity. ASL Prespectives in Logic. Cambridge University Press, Cambridge (2010)

6. Feferman, S.: A language and axioms for explicit mathematics. In: Crossley, J. (ed.) In Algebra and Logic of Lecture Notes in Mathematics, vol. 450, pp. 87-139. Springer, Berlin (1975)

7. Feferman, S.: Constructive theories of functions and classes. In: Boffa, M., Dalen, D.van , McAloon, K. (eds.) In Logic Colloquium '78, pp. 159-224. North Holland, Amsterdam (1979)

8. Ferreira, F.: Polynomial time computable arithmetic and conservative extensions. $\mathrm{PhD}$ thesis, Pennsylvania State University (1988)

9. Ferreira, F.: Polynomial time computable arithmetic. In: Sieg, W. (eds.) Logic and Computation, Proceedings of a Workshop held at Carnegie Mellon University, 1987, vol. 106 of Contemporary Mathematics. American Mathematical Society, Providence, Rhode Island, pp. 137-156 (1990)

10. Ferreira, F: Stockmeyer induction. In: Buss, S., Scott, P. (eds.) In Feasible Mathematics, pp. 161-180. Birkhäuser, Basel (1990)

11. Ferreira, F.: A note on a result of Buss concerning bounded theories and the collection scheme. Port. Math. 52(3), 331-336 (1995)

12. Jäger, G.: Iterating admissibility in proof theory. In: Logic Colloquium '81. Proceedings of the Herbrand Symposion. North Holland, Amsterdam (1982)

13. Jäger, G.: Theories for Admissible Sets: A Unifying Approach to Proof Theory. Bibliopolis, Napoli (1986)

14. Pohlers, W.: Subsystems of set theory and second order number theory. In: Buss, S.R. (eds.) In Handbook of Proof Theory, pp. 209-335, North Holland (1998)

15. Probst, D.: The provably terminating operations of the subsystem PETJ of explicit mathematics. Annal. Pure Appl. Logic (to appear)

16. Sato, K.: The strength of extensionality II—-weak weak set theories without infinity. Annal. Pure Appl. Logic 162, 579-646 (2011)

17. Sazonov, V.: On bounded set theory. In: Maria Luisa Dalla Chiara et al. (ed.) Tenth International Congress of Logic, Methodology and Philosophy of Science, Florence, August 1995, vol. 1, pp. 85-103. Kluwer, September (1997)

18. Spescha, D.: Weak systems of explicit mathematics. PhD thesis, Universität Bern, (2009) 
19. Spescha, D., Strahm, T.: Elementary explicit types and polynomial time operations. Math. Logic Quart. 55(3), 245-258 (2009)

20. Spescha, D., Strahm, T.: Realizability in weak systems of explicit mathematics. Math. Logic Quart. (to appear) 